\title{
Trabajo sustentable y saludable en un entorno cambiante: ¿Cuál es el papel y los aportes de la Salud Pública?
}

\author{
Sustainable and healthy work in a changing environment: \\ What is the role and contribution of Public Health?
}

\begin{abstract}
The scope of this article is an assessment of the relationship between work and health, at a time of changes such as globalization, the introduction of new technologies in the labor environment and an epidemiological and demographic transition, which mainly affect the economically active population. Work has an effect that goes beyond physical and mental health to include well-being, which is why it is mandatory to think about "healthy and sustainable work" and its implications. This reflection should integrate the public health perspective because of its interest in the health of the population as a whole, while rethinking the role of this field of knowledge in its interaction with other areas, especially with occupational health. It is therefore necessary to reconsider the epistemological framework of public health and occupational health. For this purpose, the following categories will be analyzed: changes in the labor environment, the impact of work on health, and healthy and sustainable work as a social response to these dynamics.
\end{abstract}

Key words Work, Public Health, Occupational Health, Epistemology
Resumen Este artículo es un ensayo acerca de la relación trabajo y salud, en un momento de cambios tales como la globalización, la introducción de nuevas tecnologías en el ambiente de trabajo y la transición epidemiológica y demográfica, que afectan principalmente a la población económicamente activa. El trabajo tiene un efecto que va más allá de la salud física y mental comprendiendo al bienestar, entonces se hace necesario pensar en un "trabajo saludable y sustentable". Una reflexión que debe traer la perspectiva de la salud pública por su interés en la salud de los colectivos, pero replanteando el papel de este campo del conocimiento en su interacción con otras disciplinas, particularmente con la salud laboral. Por tanto, hay que repensar el marco epistemológico de la salud pública y la salud laboral. Para tal fin se analizarán las siguientes categorías: cambios en el mundo del trabajo, el efecto del trabajo en la salud y el trabajo saludable y sustentable como una respuesta social ante estas dinámicas.

Palabras claves Trabajo, Salud Pública, Salud Laboral, Epistemología 


\section{Introducción}

El mundo del trabajo hoy experimenta una variedad de cambios en diferentes aspectos: tecnológicos, económicos, sociales y políticos que impactan a millones de personas, en particular de las que se encuentran en la etapa económicamente activa de la vida ${ }^{1}$. Ese cúmulo de fuerzas de cambio, junto a otras macro tendencias como el aumento en la expectativa de vida de las poblaciones y la variación en los perfiles de salud, fenómenos reconocidos por diferentes entes multilaterales ${ }^{2}$, son dinámicas que han venido y siguen transmutando esa concepción de la naturaleza del trabajo tal como emergió a partir de la revolución industrial, donde se le comprendió como un intercambio de unas horas fijas de actividades productivas realizadas con un pago en retribución, de acuerdo con la perspectiva del taylorismo ${ }^{3}$. Una idea que en años posteriores se fue haciendo más compleja al incorporar en esa representación del trabajo remunerado en dinero de manera exclusiva, beneficios adicionales como cobertura en salud, pensión, etc., que no obstante ha empezado a quedar atrás ${ }^{4}$.

El trabajo desempeña un rol clave en la salud humana por ser un factor que contribuya a la enfermedad, pero de igual forma favorecer la salud. Incluso más allá de la salud per se, este, es un aspecto de primer orden en el bienestar, tal como ha sido propuesto por el programa de las Naciones Unidas para el desarrollo humano, que recoge gran parte de los elementos de la teoría de las capacidades de Amartya Sen, la cual establece que el trabajo y el desarrollo humano debe interactuar de forma sinérgica en procura de mejores condiciones y más oportunidades ${ }^{5,6}$. Incluso considerando la participación del trabajo sobre el uso del tiempo por los seres humanos, este se extiende más allá una jornada diaria ordinaria de actividad laboral, por lo que hoy en día el concepto de "work-life balance" que al español se traduce como equilibrio entre el trabajo y vida familiar, es fundamental en ese bienestar?

En un mundo en constante cambio y con una trasformación del concepto trabajo y de las relaciones de empleo, se hace indispensable cuestionarse ¿hasta qué punto es posible lograr un trabajo sustentable y saludable en tal contexto? La propuesta de "trabajo saludable y sustentable" surge ante la emergencia de las transformaciones intrínsecas a este campo y por fuera de este. Esto sustentado en la idea ya discutida acerca de la influencia del trabajo más allá del proceso salud-enfermedad que hace que se convierta en un determinante clave del bienestar. Para dar respuesta a esta pregunta se considera pertinente analizar la interacción salud pública y la laboral ${ }^{8}$.

En consecuencia, este ensayo inicia recapitulando los cambios en el mundo del trabajo, para luego analizar los efectos del trabajo en la salud y discutir acerca del "trabajo sustentable y saludable". Abordando finalmente cuál es el rol que ha de asumir la salud pública y su interacción con otras disciplinas, en particular con la salud laboral, al abordar los retos que genera la incertidumbre sobre el futuro del trabajo. Esto amerita ser considerado debido a que el empleo y las condiciones de trabajo son determinantes claves de las inequidades en salud y socioeconómicas que afectan el bienestar laboral y extralaboral. Recalcando por supuesto que, si el trabajo puede afectar de manera negativa el bienestar, la ausencia de trabajo puede ser aún más deletérea9 .

\section{Un contexto cambiante y dinámico}

El desarrollo de la internet, la mayor facilidad de viajar alrededor del mundo, una rápida urbanización, crisis económicas, auge y caída de movimientos e ideas políticas son algunas de esas nuevas dinámicas del mundo de hoy. Dentro de estas, una de las más mencionadas ha sido la globalización, la cual puede ser entendida como el "proceso resultante en el aumento del flujo de bienes, servicios, dinero, gente, información y cultura a través de las fronteras de las naciones"10. Aunque, hay otros motores de transformación cómo los cambios tecnológicos, que merecen ser discutidos en cuanto a su influencia en la evolución del concepto del trabajo y por ende en cómo éste ha incidido en la salud de las poblaciones humanas ${ }^{11}$.

Las transformaciones que han impactado de manera contemporánea en la naturaleza del trabajo pueden ubicarse históricamente después de la posguerra, específicamente en la segunda mitad del siglo XX. Inicialmente la desindustrialización que comienza hacia finales de los sesentas en países de altos ingresos comenzó un cambio en los patrones de empleo pasando a disminuir los puestos de trabajo en las ocupaciones manuales o como se conoce en inglés "Blue-collar worker" debido a la transición de actividades económicas basadas en la manufactura al sector servicios, por cambios económicos, pero también debido a innovaciones técnicas dirigidas a reemplazar al trabajador operativo. Esto tenía el interés de cambiar el mercado laboral a ocupaciones que requerirían habilidades altamente especializadas 
con un mejor salario. No obstante, la introducción de nuevas tecnologías en el trabajo a partir de los años sesenta y setentas, no se limitó al campo de acción de esos trabajos manuales ${ }^{12}$, sino que con la llegada de las computadoras revolucionó el mundo del trabajo, en particular en lo relacionado con las tareas que realizan los denominados White-collar worker un término en inglés que hace referencia a los "trabajadores de cuello blanco" es decir a aquellos que gracias a su mayor nivel de competencias, por lo general, académicas realizan actividades de tipo administrativo. Esto desencadenaría una serie de modificaciones, que influyeron en el control de la tarea que realizaba el trabajador. De manera concreta las mejoras incrementales en el desarrollo de software y hardware liberaron a algunas ocupaciones de actividades repetitivas ${ }^{13}$. La situación previamente descrita se replica en Latinoamérica donde hay cifras que muestran una caída en el empleo en la industria y una concentración de este en el sector servicios ${ }^{14}$.

Esos avances en las tecnologías de la información (TI), han venido reconfigurando incluso al concepto de espacio de trabajo tanto temporal y físico. Lo que genera el concepto de "tecnología disruptiva”, que hace alusión a la ubicuidad de esta e implica el traspaso del plano físico del ambiente laboral, irrumpiendo en otros espacios de la vida humana ${ }^{15}$. En Latinoamérica el acceso y cobertura de las TI ha tenido un rezago, que no solamente ha impactado el desarrollo educativo sino también del trabajo, al no permitir generar empleos de calidad que le permitan insertarse de manera exitosa en la economía global de la información ${ }^{16}$. Lo paradójico, es que pesar de lo anterior, incluso hay una resistencia desde la academia regional a la tecnología; por ejemplo, el profesor Giovanni Alves, considera que estas precarizan al trabajador, lo que genera una percepción negativa al respecto ${ }^{17}$.

Las crisis, transformaciones políticas y económicas son elementos que también han moldeado hasta el día de hoy la naturaleza del trabajo. Específicamente al situar el análisis de esa evolución, en un nivel meso, es decir desde las empresas, donde las reformas que afectaron al trabajo están representadas en la introducción de prácticas que promovieron los "recortes" de las plantillas de trabajadores o como eufemísticamente se le ha denominado "restructuración" de personal. A nivel mundial a partir de la década del ochenta se consolidan estos procesos de reingeniería de las empresas, expresados de manera concreta en fusiones, alianzas estratégicas y privatizaciones fruto también del esfuerzo de las empresas por ser más competitivas ${ }^{18}$. Esta transformación de la empresa, tanto en el sector público y privado, al ser el ente por excelencia generador de empleo fue uno de los ejes de la reconfiguración del trabajo, trayendo nuevas formas de contratación laboral como subcontratación, tercerización, etc.

Los cambios que están afectando al trabajo no pueden afirmarse que provengan desde un solo origen; por el contrario, tienen diferentes fuentes las que se yuxtaponen al hacer visible su efecto. Por ejemplo, el arribo y la consolidación del internet y demás tecnologías de la información (TI), están transmutando la producción y productividad, unos avances que de acuerdo con lo propuesto por Barley, profesor de la ciencias de la gestión e ingeniería de la Universidad de Stanford ${ }^{19}$; ha sentado las bases de una nueva revolución industrial, que no sólo reconfigura las actividades laborales y la disponibilidad de empleos $^{20,21}$.

Personalmente lo que se pudiera considerar más crítico de esos cambios, es la desregularización de los contratos de empleo, al afectar principalmente lo que se denomina aún hoy en día, una relación laboral estable, un concepto más reconocido por la palabra en inglés "full time job" s en la cual el trabajador solamente estaba a las órdenes de un único empleador de tiempo completo y de manera indefinida, por lo menos hasta jubilarse. Constituyéndose en una alteración del "patrón estándar de empleo" de acuerdo a lo que planteó desde mediados de la década de los ochenta Efrén Córdova un ex directivo de la Organización Internacional del Trabajo (OIT) ${ }^{22}$.

Esta laxitud progresiva en las condiciones de empleo ha sido un fenómeno generalizado a nivel mundial, ante la cual han emergido diferentes respuestas sociales por ejemplo en Europa y en los países escandinavos, donde más cerca se está del denominado "Estado de bienestar" cuyo objetivo es prevenir la extrema pobreza y garantizar la seguridad social especialmente cuando no hay ingresos ${ }^{23}$. Se ha abordado esta situación específica mediante una propuesta denominada "flexicurity", concepto que no tiene una traducción literal adecuada al español pero que en síntesis plantea la necesidad de buscar la seguridad del trabajador en un mercado laboral que tiende a ser flexible e incierto ${ }^{24}$. En contraste, en Latinoamérica la respuesta social ante esta novedad y a otras traídas por el neoliberalismo fue la consolidación de movimientos progresistas. Los que, en algunos países de la región ganaron procesos electorales llevándolos al poder. Por lo que sería 
interesante a futuro comparar la dialéctica de estas reacciones aún en proceso de estructuración frente al devenir de esta situación.

Por último, es necesario recalcar en este apartado, las transiciones en la composición etaria de las poblaciones y la reconfiguración del perfil de salud asociado. Porque desde la perspectiva del autor del presente ensayo se juzga que es en este punto donde la salud pública puede ayudar a entender y afrontar esos retos. El aumento de la proporción de trabajadores mayores, no se limita solamente a ser una cuestión demográfica sino que va mucho más allá, puesto que muchos de esos trabajadores quieren extender su vida laboral incluso más allá de la edad de retiro por varias razones: financieras para alargar el tiempo de trabajo, por el hecho que la jubilación implica una reducción del ingreso ${ }^{25}$. Esos trabajadores buscan continuar usando sus experiencias y conocimientos disfrutando de la interacción social que ofrece el lugar de trabajo como un factor que puede promover la salud; además existe un interés de incluir a las personas mayores en el trabajo, iniciado en países con un proceso de transición demográfica más avanzado, que muy seguramente se extenderá a zonas como Latinoamérica donde ya se ha reconocido este fenómeno ${ }^{26,27}$. Ante el cual la salud pública aporta enfoques teóricos como el de la transición de la salud, propuesto por Caldwell, un demógrafo australiano y Frenk, salubrista público latinoamericano ${ }^{28}$, ya que la comprensión de las implicaciones del nuevo perfil de salud de las poblaciones permitirá mejorar su bienestar, que incluye la interacción trabajo-salud.

\section{Una cuestión fundamental: cómo el trabajo afecta la salud}

Este puede influenciar la salud especialmente de tres maneras: las condiciones del ambiente de trabajo, las condiciones de la tarea y las formas de contratación. Analizando la primera, el del impacto del ambiente físico de trabajo, es el que ha sido más ampliamente estudiado y a la vez el más regulado por las diferentes legislaciones en el mundo ${ }^{29}$. Esta visión sin duda ha sido el abordaje tradicional de la relación salud trabajo, en particular, desde el enfoque reconocido como salud ocupacional. No obstante, de su importancia y sus logros en cuanto a la generación de una normatividad que proteja la salud del trabajador ${ }^{30}$, es una visión limitada de la relación salud trabajo porque está centrada en lo que pasa especialmente en el espacio proximal de labor, desconociendo o subvalorando otros influjos de tipo más distal.
Una segunda vía por la cual el trabajo afecta la salud ha sido por medio de labores y tareas relacionadas al estrés ${ }^{31}$. Área en la que también se ha centrado una gran parte de la investigación en salud laboral, desarrollando incluso modelos teóricos para su comprensión como el de Karasek y Theorell o el propuesto por Siegrist et al. ${ }^{31,32}$. Aunque está centrado en aspectos psicosociales del trabajo, en particular de la actividad como tal y sus aspectos relacionados. Usualmente supone una evocación por lo usual negativa del trabajo sobre la salud. Esa mirada enfocada sobre los aspectos generadores de malestar en el trabajo, se piensa que afortunadamente está evolucionando a una percepción más positiva, que hace énfasis en los beneficios, el bienestar y la satisfacción con la actividad laboral de acuerdo con la propuesta de Wright \& Cropanzano, docentes del departamento de ciencias de la gestión en la Universidad de Nevada ${ }^{33}$.

A mediados de la década de los noventas se empezó a reconocer un tercer modo por el cual el trabajo tiene un impacto sobre la salud, y es el centrado en la naturaleza contractual del empleo; tema reconocido de importancia en el libro: "Psychological Contracts in Organizations Understanding Written and Unwritten Agreements" escrito por Rousseau una profesora de psicología industrial en la Universidad Carnigie Mellon ${ }^{34}$. En este se examina la susceptibilidad en la forma que se estructura el contrato laboral, que especialmente ante los periodos de crisis económicas, sufre modificaciones que a su vez afectan el bienestar de los trabajadores; convirtiéndose en un referente para las ciencias organizacionales; un campo del conocimiento, ser de interés para el salubrista público y/o laboral; en razón que estudia cómo los individuos construyen estructuras organizacionales procesos y prácticas que moldean las relaciones sociales e impactan en la vida de los individuos. Permitiendo entender a las organizaciones, uno de los marcos de referencia donde transcurre la relación salud-trabajo ${ }^{35}$. A posteriori, la propuesta de Rousseau fue corroborada de modo empírico por Quinlan ${ }^{36}$, un profesor de administración de empresas en Australia, quien estudió la restructuración del mercado laboral y su efecto en desenlaces como accidentes de trabajo y enfermedad laboral, encontrando una relación directamente proporcional ${ }^{36}$.

De acuerdo con lo anterior, esto configura un nuevo campo de investigación con una perspectiva macro en el estudio de la relación salud y trabajo. El cual debe integrarse en el constructo epistemológico no solamente de la salud laboral 
sino también de la salud pública, en aras de mejorar la comprensión de relación salud trabajo. Dado que existe un marco teórico, que relaciona el trabajo, la salud y el bienestar ${ }^{37}$, en particular desde uno de los enfoques teóricos en salud pública como es el determinantes sociales; el cual mencionan que el empleo provee los recursos económicos, y que el trabajo suple una serie de necesidades psicosociales al ofrecer un status social, hace que se considere al trabajo como un eje central del gradiente social en salud, de acuerdo a lo planteado por Siegrist et al. ${ }^{38}$. La integración de estos elementos no sólo permite pasar de la salud al bienestar sino también ampliar el objeto de estudio de la salud laboral.

\section{Discutiendo el concepto de trabajo sustentable y saludable}

El concepto de sustentabilidad tiene una historia que está ligada más a los temas relacionados con desarrollo y conservación del medio ambiente. No obstante, hay que tener en cuenta que el concepto de sustentabilidad usado en este ensayo se apoya en lo que se conoce como empleabilidad sustentable una idea presente en la agenda de la política pública del sector trabajo ${ }^{39}$. El integrar el concepto de sustentabilidad dentro de esta reflexión se debe a que este se entrelaza con el concepto del desarrollo humano previamente introducido. En este sentido la sustentabilidad se entendería en como el beneficio de contar con un trabajo y que a futuro también ${ }^{40}$.

Ahora bien, pensar en que el trabajo sea "sustentable" en un entorno globalizado puede ser un contrasentido; debido a que uno de los fenómenos acompañantes de este proceso, es la flexibilización del empleo. Concepto que evoca el desmonte del status quo en cuanto a las regulaciones del mercado laboral, lo que implicaría la eliminación en las restricciones en la contratación laboral con el fin de aumentar la eficiencia y competitividad en un entorno que así lo demandaba $^{41}$; que, si bien al principio de la década de los ochenta, donde surge el término, pudiera haber significado lo mismo, no es así según el análisis de Lagos un consultor de la $\mathrm{CEPAL}^{42}$, para quién la flexibilización es la capacidad de adaptarse a la variabilidad de la circunstancias, por lo que este término no necesariamente debe sugerir una connotación negativa.

Por otra parte, teniendo en cuenta que el periodo del tiempo en el que un ser humano está en edad de trabajar, se extiende sobre una gran parte del ciclo vital, se considera que un trabajo susten- table es aquel que se inserta en ese curso de vida de manera armónica, que implicaría lograr las siguientes transiciones relacionadas con el curso de vida laboral: del estudio al trabajo, de empleado a desempleado y el paso al retiro de la manera menos abrupta logrando mantener el equilibrio entre las diferentes esferas de la existencia. Esto significa que no debería ser traumático una vez que salga de la etapa de formación conseguir empleo como les sucede a los jóvenes no solamente de países desarrollados sino en vías de hacerlo. $\mathrm{O}$ cuando el mercado laboral tiene un escaso nivel de protección ante el desempleo lo que ocasiona que esta situación impacte de manera importante su estatus socioeconómico no solo del trabajador sino también de su núcleo familiar. Finalizando, con un paso al retiro que le permita vivir con una renta que satisfaga las necesidades básicas. Para alcanzar este balance a lo largo de la trayectoria laboral se acoge el planteamiento de Benach et al. ${ }^{43}$; en relación con el concepto de trabajo justo, es decir, que la vida laboral este enmarcada en un contexto de protección social.

Ahora al abordar la otra parte del concepto propuesto relacionada con la salud, se partió de propuestas previamente estructuradas como la Promoción de la Salud en los Lugares de Trabajo por la Organización Panamericana de la Salud (OPS), en cuanto a que se comparte la mayoría de los principios en cuanto a su abordaje holístico de la salud del trabajador ${ }^{44,45}$. La otra propuesta es la de Entornos Laborales Saludables, de la cual se toma la idea del proceso iterativo en la búsqueda del bienestar de los trabajadores ${ }^{45}$. En consecuencia, en el marco de esta reflexión ¿qué se entiende por trabajo saludable? En primera instancia se considera que es aquel que permitiría prolongar la vida laboral de las personas, en un momento en el que no solamente se demanda trabajar más tiempo para adquirir un derecho como la pensión, sino que también las personas pueden esperar trabajar más tiempo en razón de los beneficios sobre la salud de continuar activos. Este concepto debe ser comprendido no solamente como el trabajo que permita la protección contra los accidentes y enfermedades derivadas de las condiciones en las que se labora, sino también de otras condiciones que tienen un impacto sobre la salud como son las enfermedades crónicas no transmisibles, una noción que ha empezado a ser tenido en cuenta con la estrategia del Total Health Worker ${ }^{\circledast}$ por el National Institute for Occupational Safety and Health (NIOSH) ${ }^{46}$. A partir de esta reflexión, se sugiere que el trabajo como "lugar" se convierta en un entorno saludable. De esta for- 
ma se seguiría con los lineamientos de la Organización Mundial de la Salud (OMS) ${ }^{47}$, donde se promueve la salud laboral de manera integral y no fragmentada, lo cual es uno de los mensajes centrales de este ensayo. Con lo que se espera el trabajo saludable se convierte en una fuente de dignidad, satisfacción y autorrealización para las personas a lo largo del curso de sus vidas. En síntesis, un trabajo sustentable y saludable es aquel que permite disfrutar de la posibilidad de extender la vida laboral de las personas, con un ingreso adecuado y en unas condiciones de empleo que permitan disminuir las inequidades sociales que afectan la salud y el bienestar de la población.

\section{Repensando el rol de la Salud Pública y su interacción con otros campos del conocimiento}

La Salud Pública ha tenido un rol importante en el abordaje de la relación salud-trabajo, desde tiempo atrás. Puesto que su objeto de estudio se divide grosso modo en dos grandes aspectos: el estudio de las condiciones de salud de las poblaciones y la respuesta social organizada ante estas condiciones. Dentro de estos subgrupos poblacionales se encuentran los trabajadores, lo que da pie a que se incluya a la salud ocupacional dentro del amplio campo de la salud pública ${ }^{48}$. Un campo del conocimiento que parece abarcar tanto, pero a la vez se puede quedar corto en su análisis, especialmente en lo relacionado con la salud laboral. Teniendo en cuenta que la evolución en la naturaleza del trabajo con sus singularidades ha llevado a que la salud ocupacional evolucione al desarrollar un objeto del conocimiento, desde una perspectiva epistemológica, con sus propias características lo que la lleva de forma contemporánea a denominarse seguridad y salud en el trabajo $^{49}$. De tal suerte que puede considerarse que la salud laboral ya no está embebida completamente en el universo de la salud pública, al nutrirse de otras disciplinas como la ergonomía, economía, el derecho y las ciencias de la gestión u organizacionales, las cuales pueden contribuir a una mejor comprensión de los cambios que han afectado al trabajo especialmente en los últimos años y por ende dar una mejor respuesta ante ellos. Por lo que, en opinión del autor del presente ensayo, se considera que la Salud Pública debe procurar una investigación que la lleve a interactuar con otras disciplinas, no en un modo de subordinación sino por el contrario en un mismo nivel, caso concreto con la salud laboral y otros campos del conocimiento previamente mencionados que le permita superar sus limitaciones al momento de abordar la relación salud-trabajo especialmente desde la esfera de lo extra laboral, apoyando puntualmente lo que en Inglés se ha denominado "work-life balance", que al español se puede traducir como conciliación de la vida laboral y familiar, que son "Las disposiciones y prácticas de conciliación se refieren a iniciativas introducidas voluntariamente por las empresas que faciliten la conciliación de trabajo de los empleados y su vida personal" ${ }^{50}$.

\section{A modo de conclusión}

El trabajo durante las últimas décadas ha experimentado cambios en la forma de realizarlo, en cómo se establecen las relaciones de empleo, en la transformación de las fuentes de empleo de las economías basadas en la industria al sector servicios y en la progresiva desaparición de los límites espaciales y temporales donde se realiza el trabajo como actividad (por ejemplo, el teletrabajo). Es indiscutible que el trabajo tiene una profunda relación con la salud siendo un modulador de primer orden en cuanto a sus desenlaces, y a la vez tiene una estrecha conexión con otros determinantes sociales de la salud como el ingreso, estatus socioeconómico, comportamientos y estilos de vida. Es evidente que el trabajo no va a dejar de experimentar transformaciones; por tanto, ante esas fuerzas que lo moldean como la globalización la respuesta razonable es aprender a adaptarse, para insertarse en una comunidad transnacional, evitando el aislacionismo y posturas inflexible ante los desafíos del cambio. Es por esto, que esta reflexión hace eco de propuestas de la OMS y la OIT, en cuanto a que el trabajo debe ser digno, promover la salud y adaptarse a las nuevas dinámicas de un entorno altamente interconectado con justicia social.

Ahora bien, ¿hasta qué punto es posible alcanzar un trabajo sustentable y saludable?, eso depende de los trabajadores, empresarios, Estado y realmente de la comunidad internacional. Porque va a ser difícil mantener unas condiciones favorables en relación con el trabajo y la salud en algunas naciones, mientras que otras aún persis$\tan$ situaciones tan deplorables en el mercado laboral como el trabajo esclavo e infantil e incluso la alta informalidad, que afecta a Latinoamérica. También estará condicionado por el hecho de lograr puntos intermedios en la concertación de los diferentes estamentos frente a buscar un equilibrio entre la flexibilización en la contratación y la seguridad y bienestar de los trabajadores actuales 
y futuros. Finalmente, se considera que, si bien el rol de la salud pública, sigue siendo importante para la compresión de la propuesta que surge en este ensayo, este puede ser limitado, fundamentalmente si no se reconoce la importancia de otros campos del conocimiento, lo que dificulta lograr una acción no sólo interdisciplinaria sino transdiciplinaria, para abordar las metamorfosis descritas y otras que no se exploraron en este ensayo como el efecto de la inteligencia artificial y la presencia creciente de los robots en entornos laborales; ese es el gran reto, ¿será posible lograrlo?

\section{Referencias}

1. Pelfrene E, Vlerick P, Moreau M, Mak RP, Kornitzer M, Backer G. Perceptions of job insecurity and the impact of world market competition as health risks: Results from Belstress. J Occup Organ Psychol 2003; 76(4):411-425.

2. Organización Panamericana de la Salud (OPS), Organización Mundial de la Salud (OMS). Oficina Regional para las Américas. Salud en las Américas. Washington DC: OPS; 2012.

3. Guidotti TL. Taylorism, the Aging Workforce, and the Biopsychosocial Model. Arch Environ Occup Health 2011; 66(4):191-192.

4. Bridges W. Jobshift: How To Prosper In A Workplace Without Jobs. Revised ed. Reading: Da Capo Press; 1995.

5. United Nations Development Programme. $2015 \mathrm{Hu}$ man Development Report [Internet]. Geneva: UN; 2015 [cited 2016 Jul 15]. Available from: http://report. hdr.undp.org/

6. Sen A. Human Rights and Capabilities. J Hum Dev 2005; 6(2):151-166.

7. Dave J, Purohit H. Work Life Balance and Perception: a conceptual framework. Clarion- Int Multidiscip J 2016; 5(1):98-104

8. Scheneider D, Lilienfeld D, editors. Public health: the development of a discipline Volume 2, Twentieth-century challenges. New Jersey: Rutgers University Press; 2011.

9. Benach J, Muntaner C, Santana V. Employment Conditions and Health Inequalities Final Report to the WHO Commission on Social Determinants of Health (CSDH) Employment Conditions Knowledge Network (EMCONET) [Internet]. Barcelona; 2007 [cited 2016 Oct 12]. Available from: http://www.who.int/social_determinants/resources/articles/emconet_who_report.pdf

10. Guillén MF. Is Globalization Civilizing, Destructive or Feeble? A Critique of Five Key Debates in the Social Science Literature. Annu Rev Sociol 2001; 27(1):235260.

11. Castles S, Haas H de, Miller MJ. The age of migration : international population movements in the modern world. New York: The Guilford Press; 2005.
12. Kollmeyer C, Pichler F, Wolfgang O, Amelie Q-V. Is Deindustrialization Causing High Unemployment in Affluent Countries? Evidence from 16 OECD Countries, 1970-2003. Soc Forces 2013; 91(3):785-812.

13. Kellaway L. Cómo el computador cambió la oficina para siempre - BBC Mundo [Internet]. BBC; 2013 [cited 2016 Oct 7]. Available from: http://www.bbc.com/ mundo/noticias/2013/09/130926_serie_oficina_computadoras_finde_cch

14. Organización Internacional del Trabajo. Panorama Laboral 2016 de América Latina y el Caribe [Internet]. LIma; 2016 [cited 2017 Aug 17]. Available from: http://www.ilo.org/americas/publicaciones/panorama-laboral/WCMS_537803/lang--pt/index.htm

15. Christensen CM. The innovator's dilemma: the revolutionary book that will change the way you do business. New York: Harper Collins; 2003.

16. CNN Español. Estos son los países de la región con mejor y peor conexión a internet, según la CEPAL | CNNEspañol.com [Internet].2016 [cited 2017 Aug 18]. Available from: https://cnnespanol.cnn.com/2016/09/14/ estos-son-los-paises-de-la-region-con-mejor-y-peorconexion-a-internet-segun-la-cepal/

17. Alves G. Trabalho, subjetividade e capitalismo manipulatório - O novo metabolismo social do trabalho e a precarização do homem que trabalha. Rev da RET 2011; V(8):31.

18. Cooper CL, Jackson SE. Creating tomorrow's organizations: a handbook for future research in organizational behavior. Chichester, New York: Wiley; 1997.

19. Barley SR. Why the internet makes buying a car less loathsome: how technologies change role relations. Acad Manag Discov 2015; 1(1):31-60.

20. Kalleberg AL. Nonstandard Employment Relations: Part-time, Temporary and Contract Work. Annu Rev Sociol 2000; 26(1):341-365.

21. Organización para la Cooperación y el Desarrollo Económico (OECD). Implementing the OECD Jobs Strategy. Paris: OECD Publishing; 1999.

22. Cordova E. From Full-Time Wage Employment to Atypical Employment: A Major Shift in the Evolution of Labour Relations. Int Labour Rev 1986; 125(6):641-658. 
23. Eißel D, Rokicka E, Leaman J, editors. Welfare State at Risk. Cham: Springer International Publishing; 2014.

24. Wilthagen T, Tros FH. The Concept of Flexicurity: A New Approach to Regulating Employment and Labour Markets. Eur Rev Labour Res 2004; 10(2):166186.

25. Myles J. The Maturation of Canada's Retirement Income System: Income Levels, Income Inequality and Low-Income among the Elderly. Statistics Canada and Florida State University; 2000.

26. Macleod A, Obe W, Wilton P, Woodman P, Hutchings $\mathrm{P}$. Managing an ageing workforce [Internet]. London; 2010 [cited 2016 Oct 11]. Available from: http://www. cipd.co.uk/binaries/Managing_Ageing_Workforce. pdf

27. Attanasio O, Bonfatti A, Kitao S, Weber G. Global Demographic Trends, Capital Mobility, Saving and Consumption in Latin America and the Caribbean (LAC) [Internet]. Washington; 2015 [cited 2017 Aug 16]. Available from: https://publications.iadb.org/handle/11319/6945

28. Frenk J, Bobadilla J, Stern C, Frejka T, Lozano R. Elements for a theory of the health transition. Heal Transit Rev 1991; 1(1):21-38.

29. Venables K, editor. Current Topics in Occupational Epidemiology. Oxford: Oxford University Press; 2013.

30. Weill H, Hughes JM. Asbestos as a Public Health Risk: Disease and Policy. Annu Rev Public Health 1986; 7(1):171-192.

31. Karasek R, Theorell T. Healthy Work: Stress, Productivity, and the Reconstruction of Working Life. Michigan: Basic Books; 1990.

32. Siegrist J, Starke D, Chandola T, Godin I, Marmot M, Niedhammer I. The measurement of effort-reward imbalance at work: European comparisons. Soc Sci Med 2004; 58(8):1483-1499.

33. Wright TA, Cropanzano R. Psychological well-being and job satisfaction as predictors of job performance. J Occup Health Psychol 2000; 5(1):84-94.

34. Rousseau DM. Psychological contracts in organizations: understanding written and unwritten agreements. Thousand Oaks: SAGE Publications; 1995.

35. Clegg S, Bailey Russell J, editors. International Encyclopedia of Organization Studies. Los Angeles: SAGE; 2007.

36. Quinlan M. The Implications of Labour Market Restructuring in Industrialized Societies for Occupational Health and Safety. Econ Ind Democr 1999; 20(3):427-460.

37. Waddell G, Burton AK. Is work good for your health and well-being? London: TSO; 2006.

38. Siegrist J, Benach J, McKnight A, Goldblatt P, Muntaner C. Employment arrangements, work conditions and health inequalities: report on new evidence on health inequality reduction, produced by task group 2 for the Strategic review of health inequalities post 2010. London: Marmot Review; 2010.
39. van der Klink JJL, Bültmann U, Burdorf A, Schaufeli WB, Zijlstra FRH, Abma FI, Brouwer S, van der Wilt GJ. Sustainable employability--definition, conceptualization, and implications: A perspective based on the capability approach. Scand J Work Environ Health 2016; 42(1):71-79.

40. Picazzo Palencia E, Gutiérrez Garza E, Infante Bonfiglio JM, Cantú Martínez PC. La teoría del desarrollo humano y sustentable: hacia el reforzamiento de la salud como un derecho y libertad universal. Estud Soc 2011; 19(37):253-279.

41. Useche MC. La organización del trabajo en el marco de la globalización. Gac Labor Universidad del Zulia 2002; 8(1):67-78.

42. Lagos Weber RA. ¿Qué se entiende por flexibilidad del mercado de trabajo? Rev la CEPAL 1994; (54):81-95.

43. Benach J, Muntaner C, Solar O, Santana V, Quinlan M, editors. Empleo, trabajo y desigualdades en salud: una visión global. Barcelona: Icaria editorial; 2010.

44. Muñoz AI, Castro Silva E. Salud de los trabajadores. Salud los Trab. Universidad de Carabobo 2010; 18(2):141-152.

45. Organización Panamericana De La Salud (OPS). Estrategia para el fortalecimiento de la promoción de la salud en los lugares de trabajo en América Latina y el Caribe. San José: OPS; 2000.

46. Schill AL, Chosewood LC. The NIOSH Total Worker Health ${ }^{\mathrm{TM}}$ Program: An Overview. J Occup Environ Med 2013; 55(Supl. 12):S8-S11.

47. International Institute for Labour Studies. International Labour Office. Decent work: objectives and strategies. Geneva: International Labour Office; 2006.

48. Frenk J. La nueva salud pública. En: Organización Panamericana de la Salud (OPS). La crisis de la salud pública: reflexiones para el debate. Washington: OPS; 1992. p. 75-93.

49. Molano Velandia JH, Pinilla Arévalo N. De la salud ocupacional a la gestión de la seguridad y salud en el trabajo: más que semántica, una transformación del sistema general de riesgos laborales. INNOVAR Rev Ciencias Adm Soc 2013; 23(48):21-31.

50. McCarthy A, Darcy C, Grady G. Work-life balance policy and practice: Understanding line manager attitudes and behaviors. Hum Resour Manag Rev 2010; 20(2):158-167.

Artículo presentado en $06 / 05 / 2017$

Aprobado en 20/09/2018

Versión final presentada en 22/09/2018 